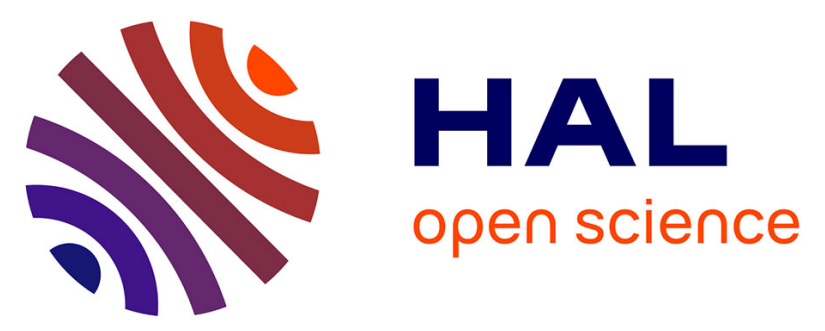

\title{
Respect des volontés en fin de vie: étude de faisabilité d'une information sur la personne de confiance et les directives anticipées
}

\author{
Pascale Vinant, Isabelle Rousseau, Olivier Huillard, François Goldwasser, \\ Marie-Yvonne Guillard, Isabelle Colombet
}

\section{To cite this version:}

Pascale Vinant, Isabelle Rousseau, Olivier Huillard, François Goldwasser, Marie-Yvonne Guillard, et al.. Respect des volontés en fin de vie: étude de faisabilité d'une information sur la personne de confiance et les directives anticipées. Bulletin du Cancer, 2015, 102 (3), pp.234-244. 10.1016/j.bulcan.2015.01.009 . hal-01372365

\section{HAL Id: hal-01372365 \\ https://hal.science/hal-01372365}

Submitted on 27 Sep 2016

HAL is a multi-disciplinary open access archive for the deposit and dissemination of scientific research documents, whether they are published or not. The documents may come from teaching and research institutions in France or abroad, or from public or private research centers.
L'archive ouverte pluridisciplinaire HAL, est destinée au dépôt et à la diffusion de documents scientifiques de niveau recherche, publiés ou non, émanant des établissements d'enseignement et de recherche français ou étrangers, des laboratoires publics ou privés.

\section{(ㅇ)(1) $\$$}

Distributed under a Creative Commons Attribution - NonCommercial - NoDerivatives 44.0 


\title{
CrossMark Respect des volontés en fin de vie : étude de faisabilité d'une information sur la personne de confiance et les directives anticipées
}

\author{
Isabelle Colombet ${ }^{1,2}$ \\ Reçu le 8 juillet 2014 \\ Accepté le 13 octobre 2014 \\ Disponible sur internet le : \\ 27 février 2015 \\ 1. AP-HP, groupe hospitalier Cochin Broca Hôtel-Dieu, unité fonctionnelle de \\ médecine palliative, 75679 Paris cedex 14, France \\ 2. Université de Paris Descartes, 75006 Paris, France \\ 3. AP-HP, groupe hospitalier Cochin Broca Hôtel-Dieu, service d'oncologie médicale, \\ 75679 Paris cedex 14, France \\ Correspondance : \\ Isabelle Colombet, hôpital Cochin, unité mobile de soins palliatifs \\ (bâtiment Copernic), 123, boulevard de Port Royal, 75679 Paris cedex 14, France. \\ isabelle.colombet@cch.aphp.fr
}

Pascale Vinant ${ }^{1}$, Isabelle Rousseau ${ }^{1,2,3}$, Olivier Huillard ${ }^{2,3}$, François Goldwasser ${ }^{2,3}$, Marie-Yvonne Guillard ${ }^{1}$,

Mots clés

Directives anticipées

Personne de confiance

Discussions anticipées

Cancer avancé

Information du patient

Préférences du patient

Keywords

Advance care planning

Advance directives

Surrogate

Advanced cancer

Patient's informed

treatment preferences

Living wills

\section{Résumé}

Cette étude prospective interventionnelle vise à montrer la faisabilité et l'impact d'une intervention d'information sur la personne de confiance (PC) et les directives anticipées (DA), pour des patients atteints de cancer pulmonaire ou digestif incurable. L'intervention comprenait deux entretiens semi-structurés réalisés en hôpital de jour d'oncologie. Le premier comprenait : recueil des souhaits sur l'information pronostique et l'implication dans les décisions, évaluation initiale des connaissances, information sur PC et DA. Le deuxième évaluait l'impact du $1^{\text {er }}$ entretien sur les connaissances, I'utilisation du dispositif, l'appréciation de l'intervention par le patient et l'évaluation de l'anxiété générée. Sur 77 patients éligibles, 23 (30\%) ont été inclus, avec un taux de refus de $21 \%$ (6/29) et un taux d'achèvement de $87 \%$ (20/23). Les patients non inclus avaient un taux de décès à 4 mois plus élevé que les inclus (39\% vs $4 \%$ ). Les patients inclus avaient une forte attente d'information et appréciaient qu'elle soit délivrée précocement, par une personne extérieure à leur prise en charge. L'étude montre la faisabilité de l'intervention et son impact sur l'utilisation du dispositif (PC et DA) par les patients, en révélant néanmoins la complexité d'une démarche d'anticipation des volontés et l'importance de l'insérer dans un processus de discussion.

\section{Summary}

Respecting patient's end of life wishes: Feasibility study of an information on surrogate and advance directives

This prospective interventional study aims to show the feasibility and impact of information procedure on surrogate and advance directives $(A D)$, for patients with incurable lung or gastrointestinal cancer. The intervention consisted of two semi-structured interviews. The first 
included: collection of preferences for prognostic information and involvement in decisionmaking, initial assessment of knowledge, information and surrogate and DA. The second assessed the impact of the first interview on knowledge, surrogate designation and DA writing, the assessment procedure by the patient and assessment of anxiety generated. Among 77 eligible patients, 23 (30\%) were included, 6/29 (21\%) refused to participate, 20/23 (87\%) completed both interviews. Patients not included had a higher 4-month death rate than included ones (39\% vs. $4 \%, \mathrm{P}=0.002$ ). Patients included had high expectations of information and appreciated it be delivered early, by someone not involved in their care. The study shows the feasibility of the procedure and its impact on the use of surrogate and DA by patients, however, revealing the complexity of approaching end-of-life wills and the importance of a process of anticipated discussion.

\section{Introduction}

En France, les cancers sont la première cause de mortalité depuis 2004. Les décisions, lorsque la maladie est à un stade avancé, sont multiples : poursuite ou non de la chimiothérapie, recours aux équipes de soins palliatifs, indication d'une alimentation parentérale, transfert en réanimation, pratique de gestes invasifs. L'encadrement législatif a mis en place plusieurs dispositifs qui peuvent favoriser au cours de ces décisions le respect de la volonté du patient en fin de vie : personne de confiance, définie dans la loi sur les droits des malades de 2002 indépendamment du contexte de fin de vie, et directives anticipées définies par la loi 2005 sur les droits des malades et la fin de vie, dite loi Léonetti). Ce renforcement du principe d'autonomie suppose une information des patients sur l'évolution de leur maladie et son pronostic. Cependant, si l'annonce du diagnostic en oncologie a fait l'objet d'un dispositif instauré par la mesure $40 \mathrm{du}$ plan cancer en 2003, l'information pronostique reste plus complexe à aborder avec une crainte de déstabiliser le patient [1]. Le dispositif légal en fin de vie est imparfaitement connu des professionnels et des patients [2,3]. Dans une étude conduite par I'INED, évaluant en France les décisions médicales en fin de vie à partir de 4723 décès, seuls 2,5\% des patients ont rédigé des directives anticipées [4]. Pourtant, les décisions médicales en fin de vie sont fréquentes ( $82 \%$ des décès) et interviennent pour deux tiers des patients à un moment où ils ne sont plus en capacité d'exprimer leurs volontés. Par ailleurs, l'étude nous apprend que la fin de vie est prévisible dans $83 \%$ des cas, toutes causes de décès confondues. En oncologie, l'existence de scores pronostiques validés [5] facilite cette prévision. L'ensemble de ces données épidémiologiques montre bien l'enjeu essentiel qu'est l'information des patients sur ces dispositifs.

Aux États-Unis, le Patient Self Determination Act a instauré, dès 1990, I'obligation d'informer toute personne admise dans un établissement public de santé, sur son droit à formuler des directives anticipées. La population américaine ne s'est cependant que progressivement approprié le dispositif, comme en témoigne l'échec de l'étude SUPPORT [6]. Plus récemment, une étude conduite, chez des patients atteints de cancer du pancréas retrouvait $15 \%$ de patients ayant rédigé des directives anticipées [7] ; dans l'étude de Lamont et al., ce résultat s'élevait à $33 \%$ dans une population de patients hospitalisés en oncologie [8]. De nombreuses études ont permis tant de définir des modalités adaptées d'information des patients sur ces dispositifs, que de comprendre les limites des directives anticipées [9]. Une revue de la littérature conclut que, pour des patients ambulatoires, l'association de discussions répétées médecinpatient à un support écrit, favorise la rédaction de directives anticipées [10]. Certains travaux fournissent également des modèles pour l'élaboration d'une intervention d'information $[11,12]$.

En France, certaines limites des directives anticipées ont été décrites : leur validité dans le temps dans le contexte d'évolution de la maladie, la vulnérabilité du patient au moment où il les écrit, les compétences du patient par rapport aux enjeux médicaux, le risque de perte de chance pour le patient [13]. À notre connaissance, aucune étude n'a évalué en France, la faisabilité et l'impact d'une information systématisée auprès de patients atteints de cancer, sur le dispositif légal encadrant les décisions médicales en fin de vie.

Cette étude prospective interventionnelle vise à explorer la faisabilité pour les patients atteints de cancer incurable, d'une intervention d'information sur les dispositifs légaux en lien avec le respect de la volonté de la personne dans les prises de décisions médicales en fin de vie : personne de confiance et directives anticipées. Nous évaluons également l'impact de cette information sur l'utilisation de ces dispositifs par les patients.

\section{Méthodes}

\section{Sujets et cadre de l'étude}

L'étude a concerné des patients hospitalisés en hôpital de jour (HDJ) du service d'oncologie du CHU de Cochin à Paris, entre le 2 août 2011 et le 9 janvier 2012. II s'agit d'une étude prospective interventionnelle de faisabilité, basée sur deux entretiens semi-structurés avec un suivi à 4 mois évaluant le statut 
vital des patients éligibles. L'ensemble des oncologues référents a donné un accord de principe pour conduire l'étude. Un avis favorable a été donné par le comité de protection des personnes lle de France 2 .

Tout patient âgé de 18 ans ou plus, atteint d'un cancer pulmonaire ou digestif, considéré comme incurable, venant consécutivement en HDJ pour une chimiothérapie ou dans le cadre d'une prise en charge palliative exclusive était considéré comme éligible. Les patients ne parlant ou ne comprenant pas le français, présentant des troubles cognitifs ou une psychopathologie identifiée dans le dossier médical, étaient non éligibles. D'autres critères de non-inclusion ont été évalués à partir de l'avis pris auprès de l'équipe pluridisciplinaire de l'HDJ (médecins, infirmier(ères) et psycho-oncologue) le jour de l'inclusion potentielle. Ces critères ont été définis a priori pour tenir compte des contraintes organisationnelles du parcours clinique du patient, et également de la vulnérabilité des patients, dans un souci de non-malfaisance : troubles cognitifs non identifiés au préalable dans le dossier médical, fragilité psychologique, contexte d'aggravation de la maladie, fin de vie. Lorsqu'un critère organisationnel empêchait l'inclusion le jour même, celle-ci était réévaluée lors des venues ultérieures du patient en HDJ. En l'absence de critère de non-inclusion, l'étude était proposée au patient par l'investigateur avec recherche de son consentement : le refus du patient constituait le dernier motif de noninclusion.

\section{Intervention}

L'intervention comporte deux entretiens à environ un mois d'intervalle, le deuxième étant fixé en fonction du programme de venue en $\mathrm{HDJ}$.

L'intervention d'information sur le dispositif légal se déroulait lors du premier entretien. Il comprenait l'évaluation :

- des acquis du patient sur la personne de confiance et les directives anticipées (connaissances et utilisation de ces dispositifs) ;

- de ses attentes en matière d'information en cas d'aggravation, et d'implication dans les décisions en fin de vie ;

- de son appréciation du caractère utile et/ou angoissant des discussions sur la fin de vie. Après cette évaluation, une brochure d'information sur les dispositifs légaux était remise et expliquée au patient, accompagnée de formulaires-type pour la désignation de la personne de confiance et l'écriture de directives anticipées.

Le deuxième entretien comprenait :

- I'évaluation de la compréhension du dispositif légal à l'aide de 10 questions (3 concernant la personne de confiance, 6 concernant les directives anticipées et 1 concernant le droit au refus de traitement) ;

- I'exploration de l'impact du premier entretien sur le choix de la personne de confiance, et la rédaction de directives anticipées ;
- I'évaluation des modalités de l'intervention par le patient. Lorsqu'un patient rapportait l'un des formulaires-type rempli, celui-ci était ajouté dans le dossier médical, selon sa volonté, et une copie était conservée avec le cahier d'observation. Ces entretiens ont été effectués par un médecin de l'équipe mobile de soins palliatifs ( $\mathrm{PV}, \mathrm{VM}$ ou $\mathrm{OH}$ ) en binôme avec une infirmière, observatrice (MYG), suivant un guide structuré, associant des questions fermées à choix multiples et des questions ouvertes. Les personnes réalisant les entretiens étaient, dans la mesure du possible, dissociées de la prise en charge clinique des patients. L'entretien se faisait en présence de l'accompagnant éventuellement présent, sauf demande explicite de celui-ci ou du patient, le parti pris étant de ne pas priver le patient d'un soutien potentiel et d'éviter une majoration de l'anxiété et une dramatisation de la démarche. En revanche, il a été demandé à la fin de chaque premier entretien si le patient souhaitait être accompagné pour le deuxième.

\section{Critère d'évaluation de la faisabilité et de l'impact de l'intervention}

La faisabilité de l'intervention a été évaluée par :

- le taux de participation par rapport à la population éligible ;

- le taux de refus ;

- la proportion de patients pour laquelle l'intervention a pu être achevée ;

- la précocité de la démarche par rapport à l'évolutivité de la maladie, appréciée au moment de l'inclusion par la mesure de l'antériorité du diagnostic, la notion d'un traitement antitumoral en cours et d'une prise en charge par l'équipe mobile de soins palliatifs.

L'évaluation a été complétée à 4 mois après l'inclusion, d'une recherche du statut vital.

Deux objectifs secondaires de l'étude ont été également évalués :

- l'impact de l'intervention d'information sur les connaissances des patients (évaluées par 10 questions notées 1 point chacune), et l'utilisation des dispositifs par les patients au moment du $2^{\mathrm{e}}$ entretien (désignation de leur personne de confiance et rédaction de leurs directives anticipées) ;

- l'évaluation des modalités de l'intervention d'information par les patients (à quel moment, par qui, systématique ou non ?), associée à un recueil qualitatif des thèmes afférents au sujet, spontanément abordés par les patients, et par la mesure de l'anxiété générée à l'issue du $1^{\mathrm{er}}$ entretien (hétéro-évaluation par l'investigateur à la fin du $1^{\text {er }}$ entretien, et auto-évaluation au début du $2^{\mathrm{e}}$ entretien, sur une échelle numérique de 1 à 10).

\section{Autres données collectées}

Certaines caractéristiques des patients ont été recueillies au début du 1e entretien : âge, sexe, mode de vie, niveau d'étude, ressources spirituelles, souhait des patients en matière d'information en cas d'aggravation et d'implication dans les décisions 
médicales. Les données en lien avec la maladie ont été collectées à partir du dossier médical : localisation de la tumeur primitive, antériorité du diagnostic, nombre de lignes de chimiothérapie, statut métastatique ou progression loco-régionale.

\section{Analyse}

Pour analyser l'impact de l'intervention et son évaluation à travers le verbatim des patients, nous avons défini trois catégories de patients en fonction de leurs souhaits concernant l'information en cas d'aggravation et d'implication dans les prises de décisions médicales en fin de vie. Les patients du " groupe I " souhaitaient être impliqués dans toutes les décisions médicales en fin de vie et être informés de manière systématique ou à leur demande en cas d'aggravation de leur maladie. Ceux du groupe Il désiraient une information systématique en cas d'aggravation de leur maladie mais peu ou pas d'implication dans les décisions médicales en fin de vie. Dans le groupe III, les patients désiraient laisser le médecin juge de l'information devant être délivrée et ne souhaitaient pas ou peu s'impliquer dans les prises de décisions médicales en fin de vie.

L'impact de l'information donnée sur l'utilisation des dispositifs par les patients a été évalué à $0,1,2$, ou 3, selon la modification entre $1^{\mathrm{e}}$ et $2^{\mathrm{e}}$ entretien d'une ou plusieurs des variables: désignation de la $P C$, évocation des souhaits concernant sa fin de vie, et rédaction de DA.
L'analyse du verbatim des patients a été conduite par 2 investigateurs indépendants (PV et IR), jusqu'à saturation des thèmes spontanément évoqués lors de l'évaluation de l'intervention par les questions ouvertes : " comment avez-vous vécu notre premier entretien » et " à l'issue de ce $2^{\mathrm{e}}$ entretien, avez-vous des remarques ou des questions que vous souhaitez aborder ».

\section{Résultats}

\section{Caractéristiques des patients inclus et faisabilité de I'intervention d'information}

Sur 106 patients atteints de cancer du poumon ou de cancer digestif, et s'étant présentés consécutivement en HDJ d'oncologie, 77 patients satisfaisaient les critères d'éligibilité, parmi lesquels 48 ont été évalués avec des critères de non-inclusion, détaillés sur la figure $1: 25$ récusés par les psychologues ou l'équipe soignante pour motif de fragilité psychologique, 6 pour aggravation et évolution en phase terminale, 2 pour troubles cognitifs, 12 du fait de problèmes organisationnels, 3 du fait de problème médical. Parmi les 29 autres patients, auxquels l'intervention d'information a été proposée, 6 ont refusé de participer et 23 ont été inclus, 14 dès la première évaluation, 9 après réitération de celle-ci lors des venues suivantes en HDJ. Les caractéristiques des patients inclus, sont présentées dans le tableau I. Les souhaits d'information des patients, interrogés en

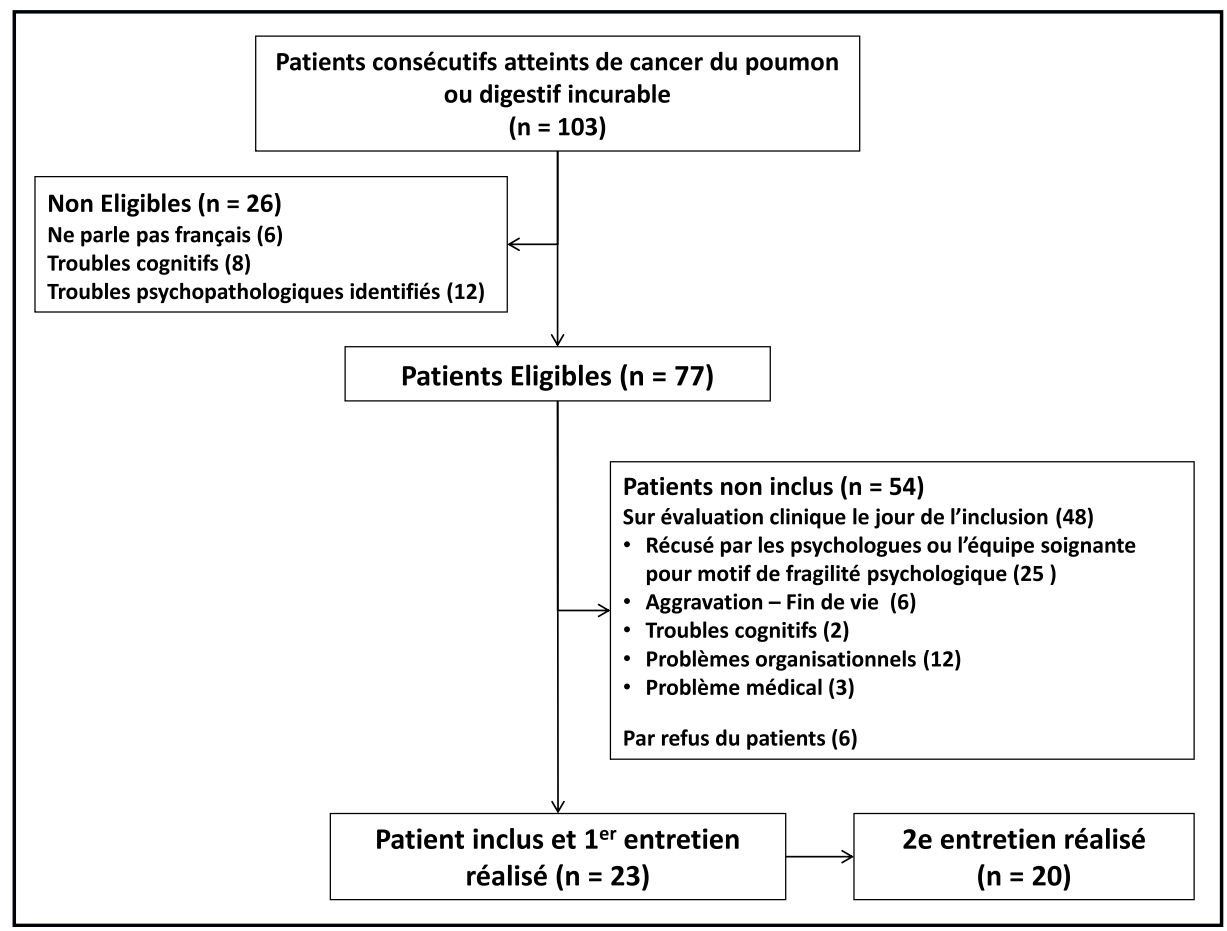

FIGURE 1

Diagramme de flux des patients dans l'étude 
TABLEAU I

Caractéristiques des patients inclus, au moment de l'évaluation initiale

\begin{tabular}{|c|c|c|}
\hline \multirow[b]{2}{*}{ Âge, moyenne (écart-type) } & \multicolumn{2}{|c|}{ Patients inclus $(n=23)$} \\
\hline & 64 & $(11,1)$ \\
\hline Hommes, $n(\%)$ & 12 & $(52,2)$ \\
\hline Vit seul $^{1}$ & 10 & $(43)$ \\
\hline \multicolumn{3}{|l|}{ Niveau d'étude ${ }^{1}$} \\
\hline Études supérieures & 15 & $(65)$ \\
\hline Collège ou lycée & 4 & $(17,4)$ \\
\hline École primaire ou pas d'études & 4 & $(17,4)$ \\
\hline Ressources spirituelles $^{1}$ & 11 & $(47,8)$ \\
\hline Dont religion : catholique & 6 & $(26,1)$ \\
\hline Dont religion : musulmane & 1 & $(4,3)$ \\
\hline \multicolumn{3}{|l|}{ Localisation tumorale primitive, $n(\%)$} \\
\hline Digestive & 9 & $(39,1)$ \\
\hline Pulmonaire & 14 & $(60,6)$ \\
\hline $\begin{array}{l}\text { Antériorité du diagnostic (mois), } \\
\text { moyenne (écart-type) }\end{array}$ & 27,3 & $(29)$ \\
\hline $\begin{array}{l}\text { Nombre de lignes de chimiothérapie, } \\
\text { moyenne (écart-type) }\end{array}$ & 2 & $(1,3)$ \\
\hline Statut métastatique, $n(\%)$ & 21 & $(91,3)$ \\
\hline Progression loco-régionale, $n$ (\%) & 14 & $(61)$ \\
\hline Traitement anti-tumoral en cours & 22 & $(95,7)$ \\
\hline Prise en charge par l'EMSP & 4 & $(17,4)$ \\
\hline Patient décédé, à l'issue de 4 mois de suivi & 1 & $(4,3)$ \\
\hline
\end{tabular}

${ }^{1}$ Questions posées au patient en début du $1^{\mathrm{e}}$ entretien.

début du 1e entretien sont présentés dans le tableau II : une majorité $(14 / 22,64 \%)$ de patients souhaite une information pronostique et être impliqués dans les décisions (groupe I), alors que seulement 2/22 (9\%) n'ont pas ce souhait (groupe III) et $6 / 22(27 \%)$ ont une position intermédiaire (groupe II). Interrogés a priori, au début du premier entretien sur leur perception de discussions anticipées sur la fin de vie, 90,5\% les pensent utiles, $85,7 \%$ les trouvent rassurantes, $54,5 \%$ des patients les trouvent angoissantes.

Le deuxième entretien a pu être réalisé pour 20 des 23 patients inclus, entre 13 et 119 jours (47 jours en moyenne) après le premier. Parmi les 3 patients n'ayant pas eu de $2^{\mathrm{e}}$ entretien, un n'est pas allé au terme du premier entretien du fait d'une anxiété croissante et a été exclu de l'étude, un patient a présenté des complications somatiques accompagnées d'angoisse rendant le $2^{\mathrm{e}}$ entretien impossible et pour le troisième, il s'est
TABLEAU II

Attentes ou conceptions des patients quant à l'information pronostique et à l'implication dans les décisions médicales

\begin{tabular}{|c|c|c|c|c|}
\hline & \multicolumn{4}{|c|}{ Souhait d'implication dans les décisions ${ }^{1}$ Tota } \\
\hline & Patient & Médecin & $\begin{array}{c}\text { Position } \\
\text { intermédiaire }\end{array}$ & \\
\hline \multicolumn{5}{|l|}{$\begin{array}{l}\text { Attentes d'information } \\
\text { pronostique }^{2}\end{array}$} \\
\hline Systématique & 13 & 4 & 2 & 19 \\
\hline Initiative du patient & 1 & & & 1 \\
\hline Initiative du médecin & & 1 & 1 & 2 \\
\hline Total & 14 & 5 & 3 & 22 \\
\hline
\end{tabular}

${ }^{1}$ Question : « Nous souhaiterions connaître votre opinion sur l'implication du patient dans les décisions médicales en fin de vie : (1) Je souhaite participer à toutes les décisions ; (2) Je fais confiance à mon médecin pour prendre les décisions nécessaires ; (3) Je me situe entre ces deux positions".

2Question: " Concernant l'information en cas d'aggravation de votre maladie, cochez la proposition qui vous convient : (1) Être informé de manière systématique ; (2) Recevoir les informations uniquement si vous le demandez ; (3) Laisser le médecin décider ce qu'il doit me dire ; (4) Ne pas être informé ».

agi d'un problème organisationnel. Aucun patient n'a refusé le deuxième entretien. La recherche du statut vital, 4 mois après l'inclusion a montré qu' $1 / 23$ patients inclus était décédé, alors que ce chiffre dans le groupe de patients éligibles non inclus était de 21/54.

Au total, l'ensemble de ces effectifs permet une estimation de la faisabilité de l'intervention d'information, avec un taux de refus de $21 \%(6 / 29)$, un taux de participation de $30 \%(23 / 77)$ et une proportion de $87 \%(20 / 23)$ de patients ayant suivi l'intervention jusqu'à son terme (cf. figure 1).

\section{Impact de l'intervention d'information Connaissances des patients}

Sur les 20 patients ayant répondu au questionnaire d'évaluation de leurs connaissances en fin du $2^{\mathrm{e}}$ entretien, dix-neuf (95\%) ont obtenu plus de $7 / 10$ bonnes réponses, (minimum 4/10, maximum 10/10), avec une moyenne à 7,8/10 (écart-type $1,23)$. Les deux erreurs les plus fréquentes rencontrées portaient sur la distinction entre personne de confiance et personne à prévenir et sur le fait que les directives anticipées ne sont pas contraignantes pour le médecin.

\section{Personne de confiance et rédaction de directives anticipées}

Au début du $1^{\text {er }}$ entretien, 11 patients définissaient correctement la personne de confiance et 10 parmi eux en avaient désigné une. Parmi les 12 patients qui ne donnaient pas de définition correcte de la $P C$, 5 déclaraient néanmoins en avoir désigné une, confondant souvent son rôle avec celui de la " personne à prévenir ". Six patients n'avaient ni désigné de $\mathrm{PC}$, ni déjà évoqué leurs souhaits et volontés en cas 
TABleAu III

Impact de la procédure chez les patients ayant un profil autonomiste (souhait d'information systématique et d'implication dans les décisions)

\begin{tabular}{|c|c|c|c|c|c|c|c|c|}
\hline \multirow[b]{2}{*}{13 patients } & \multicolumn{4}{|c|}{$1^{\text {er }}$ entretien } & \multicolumn{3}{|c|}{$2^{\mathrm{e}}$ entretien } & \multirow[t]{2}{*}{ Impact } \\
\hline & $\begin{array}{l}\text { Définit } \\
\text { la PC }\end{array}$ & $\begin{array}{l}\text { A désigné } \\
\text { sa PC }\end{array}$ & $\begin{array}{l}\text { A évoqué } \\
\text { ses souhaits }\end{array}$ & $\begin{array}{l}\text { A rédigé } \\
\text { ses DA }\end{array}$ & $\begin{array}{l}\text { A désigné } \\
\text { sa } \mathrm{PC}\end{array}$ & $\begin{array}{l}\text { A évoqué } \\
\text { ses souhaits }\end{array}$ & $\begin{array}{c}\text { A rédigé } \\
\text { ses DA }\end{array}$ & \\
\hline A & Oui & Oui & Oui & Non & Oui & Oui & Oui & 1 \\
\hline L & Oui & Oui & Oui & Non & Oui & Oui & Oui & 1 \\
\hline$T$ & Oui & Oui & Oui & Non & Oui & Oui & Oui & 1 \\
\hline U & Oui & Oui & Oui & Non & Oui & Oui & Oui & 1 \\
\hline$J$ & Oui & Oui & Oui & Non & Oui & Oui & Oui & 1 \\
\hline E & Oui & Oui & Non & Non & Oui & Non & Non & 0 \\
\hline$F$ & Oui & Oui & Oui & Non & Oui & Oui & Non & 0 \\
\hline$Q$ & Oui & Oui & Oui & Non & Oui & Oui & Non & 0 \\
\hline H & Non & Non & Non & Non & Oui & Oui & Oui & 3 \\
\hline $\mathrm{K}$ & Non & Non & Non & Non & Oui & Oui & Oui & 3 \\
\hline 1 & Oui & Non & Non & Non & Non & Oui & Oui & 2 \\
\hline D & Non & Non & Non & Non & Non & Non & Non & 0 \\
\hline$P$ & Non & Oui & Oui & Non & Oui & Non & Non & 0 \\
\hline Total de oui & $8(62 \%)$ & $9(69 \%)$ & $8(62 \%)$ & 0 & $11(85 \%)$ & $7(54 \%)$ & $8(62 \%)$ & \\
\hline
\end{tabular}

${ }^{1} L^{\prime}$ impact est évalué à $0,1,2$, ou 3, selon la modification entre $1^{\mathrm{e}}$ et $2^{\mathrm{e}}$ entretien d'une ou plusieurs des variables suivantes : désignation de la personne de confiance, l'évocation des souhaits et la rédaction des DA.

d'aggravation de leur état de santé. Parmi les 11 patients qui avaient évoqué leurs souhaits, 1 seul en avait parlé à son oncologue référent. Concernant les DA, 3 patients seulement déclaraient connaître ce dispositif et aucun n'en avaient rédigé. L'évolution entre les 2 entretiens de l'utilisation par les patients du dispositif a permis d'évaluer un impact de la procédure pour 12 patients sur les 20 ayant eu un 2e entretien. L'analyse de cet impact dans les 3 groupes de patients définis selon leurs souhaits d'information et d'implication dans les décisions, est présentée dans les tableaux III-V, ainsi que l'évaluation de l'intervention par les patients à travers leur verbatim, dans les encadrés 1-3.

Les thèmes ressortant du verbatim des patients sont : la référence à une expérience personnelle ou familiale permettant la projection dans une situation de fin de vie, la conscience de la finitude et la philosophie de vie, la difficulté de se projeter dans l'avenir, un mécanisme de mise à distance protectrice vis-à-vis de l'évocation de la fin de vie, parfois jusqu'à l'irruption d'une pensée magique, la nécessité du consentement de la personne de confiance pour son rôle de dépositaire de la volonté de la personne, le poids des décisions sur la famille et la personne de confiance et le risque de conflits familiaux, l'importance des discussions anticipées avec les médecins et de leur réitération régulière, l'insuffisance ressentie de compétences du patient pour participer aux décisions.

Au total, pour l'ensemble des patients, on peut estimer que l'intervention, lorsqu'elle a pu être conduite jusqu'au bout et évaluée par le $2^{\mathrm{e}}$ entretien, a eu un impact sur la réflexion pour 13/20 (65 \%) patients. Parmi les 7 autres patients, 3 ont préféré remettre la réflexion à plus tard, 1 ne souhaitait pas l'entamer, et 3 avaient déjà exprimé leurs choix.

\section{Mesure de l'anxiété et évaluation de l'intervention par les patients}

Pour 19 des 20 patients ayant eu les deux entretiens, I'hétéroévaluation de l'anxiété donnée par l'investigateur à la fin du premier entretien a été supérieure à l'évaluation donnée par le patient au cours du deuxième entretien. En comptant, pour chaque point d'échelle numérique, comme différence positive une surévaluation et comme différence négative une sousévaluation, nous aboutissons à un écart moyen de 1,4 points avec des extrêmes à $-2,5$ et 5 points.

Deux patients auraient préféré que l'information soit effectuée par un autre professionnel de santé : I'oncologue. A contrario, plusieurs s'accordent à dire que la personne devant donner cette information ne doit pas être impliquée dans sa prise en charge : 
TABLEAU IV

Impact de la procédure chez les patients ayant un profil intermédiaire (souhait d'information systématique ou à l'initiative du patient, décision partagée ou laissée au médecin)

\begin{tabular}{|c|c|c|c|c|c|c|c|c|}
\hline \multirow[b]{2}{*}{8 patients } & \multicolumn{4}{|c|}{$1^{\mathrm{er}}$ entretien } & \multicolumn{3}{|c|}{$2^{\mathrm{e}}$ entretien ${ }^{2}$} & \multirow[t]{2}{*}{ Impact } \\
\hline & $\begin{array}{l}\text { Définit } \\
\text { la PC }\end{array}$ & $\begin{array}{c}\text { A désigné } \\
\text { sa } P C\end{array}$ & $\begin{array}{l}\text { A évoqué } \\
\text { ses souhaits }\end{array}$ & $\begin{array}{c}\text { A rédigé } \\
\text { ses DA }\end{array}$ & $\begin{array}{l}\text { A désigné } \\
\text { sa } P C\end{array}$ & $\begin{array}{l}\text { A évoqué } \\
\text { ses souhaits }\end{array}$ & $\begin{array}{c}\text { A rédigé } \\
\text { ses DA }\end{array}$ & \\
\hline C & Non & Oui & Non & Non & Oui & Non & Non & 0 \\
\hline G & Non & Non & Oui & Non & Non & Oui & Non & 0 \\
\hline $\mathrm{N}^{2}$ & Non & Non & Non & Non & - & - & - & - \\
\hline 0 & Non & Non & Non & Non & Non & Non & Oui & 1 \\
\hline R & Oui & Oui & Oui & Non & Oui & Oui & Non & 0 \\
\hline$S$ & Oui & Oui & Non & Non & Oui & Oui & Oui & 2 \\
\hline$v^{2}$ & Non & Oui & - & Non & - & - & - & - \\
\hline $\mathrm{w}^{2}$ & Non & Oui & Oui & Non & - & - & - & - \\
\hline Total de oui & 2 & 5 & 3 & 0 & 3 & 3 & 2 & \\
\hline
\end{tabular}

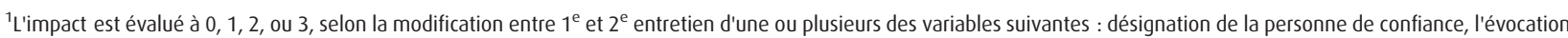
des souhaits et la rédaction des DA.

${ }^{2} 2^{\mathrm{e}}$ entretien non réalisé pour 3 patients $(\mathrm{N}, \mathrm{V}, \mathrm{W})$.

« il est important d'établir une distinction entre les personnes impliquées dans les soins et les personnes de l'anticipation : entre les soins et les choses de prévision " (Me J). D'autres insistent sur la compétence nécessaire du professionnel vis-àvis des dispositifs légaux et dans le domaine communicationnel. Le bon moment pour informer les patients de ces dispositifs de la loi est pour 11 patients, " en bonne santé », et pour 9, " au début du traitement d'une maladie grave. » Aucun patient n'a choisi les propositions « lorsque les traitements de la maladie ne sont plus efficaces » ou " à l'approche de la fin de la vie ». Seize patients sur 19 estiment que cette information doit être effectuée de manière systématique. Quelques-uns soulignent le caractère potentiellement déstabilisant de l'information donnée et l'importance de la présence de la personne de confiance.

\section{Discussion et conclusion}

Nos résultats démontrent la faisabilité, au sein d'un hôpital de jour d'oncologie, d'une information sur les dispositifs légaux permettant le respect des volontés en fin de vie, délivrée à des patients atteints de cancer incurable, après exclusion des patients estimés vulnérables. L'intervention élaborée pour l'étude a été réalisable (conduite jusqu'au bout chez $87 \%$ des patients inclus) et jugée nécessaire par une grande majorité des patients. L'information a eu un impact sur les connaissances des patients et sur leur utilisation des dispositifs de la PC et les DA. Cependant, I'analyse du verbatim des patients et la diversité des postures quant à l'implication dans les décisions médicales révèle la complexité du processus d'anticipation de la fin de vie. Le choix de ne pas inclure les patients estimés trop vulnérables à l'issue de l'évaluation initiale ne permet pas la généralisation

TABLEAU V

Impact de la procédure chez les patients ayant un profil paternaliste (souhait d'information laissée à l'initiative du médecins et décision laissée au médecin)

\begin{tabular}{|c|c|c|c|c|c|c|c|c|}
\hline 2 patients & $\begin{array}{l}\text { Définit } \\
\text { la PC }\end{array}$ & $\begin{array}{c}\text { A désigné } \\
\text { sa } P C\end{array}$ & $\begin{array}{l}\text { A évoqué } \\
\text { ses souhaits }\end{array}$ & $\begin{array}{l}\text { A rédigé } \\
\text { ses DA }\end{array}$ & $\begin{array}{l}\text { A désigné } \\
\text { sa PC }\end{array}$ & $\begin{array}{l}\text { A évoqué } \\
\text { ses souhaits }\end{array}$ & $\begin{array}{c}\text { A rédigé } \\
\text { ses DA }\end{array}$ & Impact $^{1}$ \\
\hline B & Non & Oui & Non & Non & Oui & Oui & Oui & 2 \\
\hline M & Non & Non & Non & Non & Oui & Oui & Non & 2 \\
\hline
\end{tabular}

${ }^{1}$ L'impact est évalué à $0,1,2$, ou 3, selon la modification entre $1^{\mathrm{e}}$ et $2^{\mathrm{e}}$ entretien d'une ou plusieurs des variables suivantes : désignation de la personne de confiance, l'évocation des souhaits et la rédaction des DA. 


\section{ENCADRÉ 1}

Verbatim et thèmes abordés par les patients ayant un profil autonomiste (souhait d'information systématique et d'implication dans les décisions) ${ }^{1}$

Patients pour lesquels la procédure a eu un impact : Me A : Fait référence à des expériences soit personnelle (localisation cérébrale de sa maladie) soit dans son entourage (un ami en état végétatif) qui lui a permis d'anticiper la perte de ses facultés. A conscience du poids des décisions sur la famille et souligne l'importance du consentement de la PC.

Me J : Souhaite ne pas faire peser sur autrui (sa famille) des " choses trop lourdes ». " Je souhaite vous dire quelques chose en conclusion : les fées et les lutins nous envient à cause de notre mortalité ».

Me L : Son expérience personnelle d'aphasie (métastase cérébrale) l'a déjà conduite à interpeler les médecins sur ses souhaits. C'est « une démarche rassurante et qui n'est pas antinomique avec le fait de garder l'espoir ».

Me T : Les DA sont utilisées pour renforcer le pouvoir de la PC. "Nous avons longuement parlé avec ma PC de mon cas et elle sera en mesure, si je ne le peux, de prendre les décisions nécessaires, après avoir pris connaissance de l'avis des médecins ». Mr H : Patient qui souhaite décéder dans son pays d'origine. Idées claires sur ce qui est important pour lui en fin de vie « La vie, c'est la communication, aucun traitement si plus de communication ". En revanche, la formalisation des DA se traduit simplement par "pas d'acharnement thérapeutique ".

Me I : " Ce qui est important c'est de pouvoir travailler ces questions et échanger avec les autres ", " cette démarche est à faire de manière répétée car la maladie peut progresser et on peut avoir changé d'idée ».

Mr K : « Tout le monde ne peut pas le supporter (de recevoir l'information) ; il faut une philosophie de la vie, j'ai eu une vie heureuse ».

Patients pour lesquels la procédure n'a pas eu d'impact : Me $D$ : Mise à distance défensive de l'information, " n'y a pas trop pensé, a repeint (sa) cuisine et perdu les documents». Mr P : A besoin de dire plusieurs fois au cours de l'entretien qu'il " va bien ", il lui faut se convaincre que le fait de parler de sa fin de vie ne va pas le rapprocher de l'échéance.

Mr E : Philosophie de vie acquise à l'issue d'une expérience personnelle de menace vitale étant jeune, " je me laisse emporter pas le destin » : il apprécie la démarche mais n'en a pas besoin. "Savoir que l'on va mourir, cela ne prend pas trop de place dans la tête ».

Mr Q : Réserve sur le moment des DA, « quand on n'est pas malade, on ne mesure pas la portée de ce que l'on dit, sauf si on a une expérience avec la famille, c'est plus réaliste si on est malade, mais alors, c'est démoralisant ».

Me F : Valeur d'une expérience d'acharnement thérapeutique vécue chez un proche " ces discussions sont importantes pour la dignité de la personne ".

${ }^{1}$ Verbatims en réponse aux questions ouvertes lors du $2^{\mathrm{e}}$ entretien : " comment avez-vous vécu notre premier entretien » et " à l'issue de ce $2^{\mathrm{e}}$ entretien, avez-vous des remarques ou des questions que vous souhaitiez aborder.
ENCADRÉ 2

Verbatim et thèmes abordés par les patients ayant un profil intermédiaire (souhait d'information systématique ou à l'initiative du patient, décision partagée ou laissée au médecin) ${ }^{1}$

Patients pour lesquels la procédure a eu un impact.

Me $S$ : La $P C$ vit très mal la maladie, " je veux que mes volontés soient consultées, avant ma PC », « je ne veux pas vivre mal, incontinente, inconsciente », DA rédigées et contributives. Me R : Est avocate, elle exprime la difficulté du passage à l'écrit, évoque le risque de conflits familiaux et souligne l'importance de discussions anticipées avec les médecins et la PC.

Me 0 : Souhait de suicide au début d'une autre maladie grave, pour laquelle elle s'est adaptée. A adopté depuis un fonctionnement " au jour le jour » " on verra bien ». " Est-ce qu'aujourd'hui on est en capacité de savoir ce qu'on voudrait si on était en fin de vie ? ". Au $2^{\mathrm{e}}$ entretien, elle se souvient, en voyant l'investigateur, de "la personne qui parle de la mort». Patients pour lesquels la procédure n'a pas eu d'impact : Me $\mathrm{G}$ : Ne veut pas faire peser sur son fils d'éventuelles décisions, mais la PC ne peut être quelqu'un d'autre que lui ; le principe de la PC lui pose donc problème. Fait confiance aux médecins et souhaitent qu'ils l'aident jusqu'au bout. Elle est néanmoins claire sur ce qu'elle veut (expérience de référence avec sa mère), « en parler cela fait du bien ». Elle souhaite le partager avec le corps médical. Les discussions anticipées seraient pour elles appropriées, qu'elles soient suivies ou non de DA.

Mr C : Estime n'avoir pas suffisamment de compétences pour participer aux décisions. "Si les gens se sentent moins bien après qu'avant (la procédure d'information), cela sert à quoi ?". Mr $N$ : « Je fais confiance, car je n'ai pas le choix, car je veux guérir, enfin ... être prolongé ». Le $2^{\mathrm{e}}$ entretien n'a pas lieu en raison d'une progression de la maladie et d'une angoisse très forte du patient.

${ }^{1}$ Verbatims en réponse aux questions ouvertes lors du $2^{\mathrm{e}}$ entretien : " comment avez-vous vécu notre premier entretien » et « à l'issue de ce $2^{\mathrm{e}}$ entretien, avez-vous des remarques ou des questions que vous souhaitiez aborder.

\section{ENCADRÉ 3}

Verbatim et thèmes abordés par les patients ayant un profil paternaliste (souhait d'information laissée à l'initiative du médecins et décision laissée au médecin) ${ }^{1}$

Me B : Information anxiogène et dispositif inapproprié qui viennent bousculer un mécanisme de dénégation : "Je ne m'imagine pas que j'ai cette maladie ». "Se projeter dans une situation négative est angoissant ». Son mari souligne l'importance de la présence de la PC à l'entretien.

Mr $M$ : Mécanisme de défense opérant de mise à distance : " on verra cela plus tard ».

${ }^{1}$ Verbatims en réponse aux questions ouvertes lors du $2^{\mathrm{e}}$ entretien : " comment avez-vous vécu notre premier entretien » et " à l'issue de ce $2^{\mathrm{e}}$ entretien, avez-vous des remarques ou des questions que vous souhaitiez aborder. 
des résultats de cette étude exploratoire à l'ensemble d'une population de patients atteints de cancer incurable. Le taux de participation (30\%) et le taux de refus (21\%) de notre étude, sont difficiles à comparer à ceux retrouvés dans des études portant sur la même thématique, mais dans des contextes différents. Dow et al., en proposant à des patients hospitalisés consécutivement en oncologie, un entretien semi-structuré sur les DA et le souhait de les discuter, obtiennent un taux de refus de $12 \%$ [14]. Dans une étude conduite dans un hôpital général, et évaluant, au cours d'un entretien structuré, la compréhension par les patients hospitalisés de l'information reçue à l'admission sur les directives anticipées et les manœuvres de réanimation cardio-respiratoire, le taux de participation obtenu est de $50 \%$ et le taux de refus est de $18 \%$ [15].

Dans notre étude, deux types de facteurs sont à l'origine du faible taux d'inclusion. D'une part, les contraintes organisationnelles inhérentes au fonctionnement d'un hôpital de jour ont empêché la réalisation des entretiens pour $16 \%$ des patients éligibles. D'autre part, la précaution de ne pas inclure les patients jugés trop vulnérables pour recevoir l'information délivrée sur la base d'une évaluation psychologique et soignante, constitue près de $50 \%$ des raisons de non-inclusion. Compte-tenu de la fréquence de l'anxiété, de la dépression, et des troubles de l'adaptation chez les patients atteints de cancer [16], ce parti pris de ne pas risquer de déstabiliser psychiquement le patient nous a paru justifié dans le cadre d'une étude exploratoire. Dans cette population, l'évaluation des souhaits des patients, au début du $1^{\mathrm{e}}$ entretien, a montré une forte attente d'information en cas d'aggravation et un souhait majoritaire d'implication dans les décisions. Dans une population moins sélectionnée de patients atteints de cancer au diagnostic d'incurabilité, Hagerty et al. rapportent que seuls $11 \%$ des patients préfèrent ne jamais discuter de la fin de vie [17]. Dans le contexte français, une étude conduite dans plusieurs services gériatriques retrouve également que $77 \%$ des patients souhaiteraient être informés exhaustivement en cas de situation médicale menaçant leur vie [18].

Si les patients ont en général bien compris le sujet, comme en témoigne l'impact de l'intervention sur leurs connaissances, la disparité de l'utilisation des dispositifs légaux révèle la complexité du processus d'anticipation des souhaits en fin de vie. La personne de confiance est souvent désignée, avec parfois une omission de son rôle de dépositaire de la volonté du patient, notamment dans le groupe des patients ayant un profil non autonomiste. Le rappel de ce rôle au cours de l'intervention a permis à certains patients d'initier une discussion avec celle-ci, ou de modifier leur choix, pour la protéger du poids de décisions difficiles. Dans les trois groupes de patients, les directives anticipées sont peu connues initialement, et diversement utilisés en réaction à l'information reçue. Cette différence dans l'appropriation des deux dispositifs est en lien avec l'antériorité législative de la PC [19] par rapport à celle des DA [20], et à une information plus systématique des patients sur la PC.

Le verbatim des patients révèle plusieurs facteurs pouvant freiner ou au contraire faciliter l'utilisation du dispositif légal. Pour les patients du groupe I, la capacité de la personne à se projeter dans une situation de fin de vie est un facteur facilitant, soit du fait d'une expérience personnelle ou familiale, soit du fait d'une posture existentielle acceptant la finitude. Comme le dit Me J «les fées et les lutins nous envient à cause de notre mortalité ». La conscience du poids des décisions déléguées aux proches peut autant motiver une réflexion sur le choix de la PC et une discussion avec elle que freiner sa désignation. Ce facteur, objectivé dans un contexte de réanimation [21] est également facilitateur pour la rédaction de directives anticipées qui est alors vécue comme un moyen de soulager les proches.

La démarche d'anticipation est limitée par la difficulté de se projeter dans l'avenir. Si elle fait sens pour certaines personnes qui aiment exercer un contrôle sur leur vie, pour d'autres en revanche, elle va à l'encontre d'une philosophie de vie centrée sur l'ici et maintenant : " Je me laisse porter par le destin » $(\mathrm{Mr}$ E). La limite temporelle inhérente au dispositif est exprimée par Me 0 : «Est-ce qu'aujourd'hui on est en capacité de savoir ce qu'on voudrait si on était en fin de vie ». En effet, les capacités d'adaptation des patients à chaque étape de la maladie peuvent parfois les amener à faire des choix qu'ils n'auraient pas imaginé pouvoir faire.

La crainte de l'angoisse induite par l'information freine les professionnels pour débuter ces entretiens, alors que plusieurs auteurs rapportent que les discussions anticipées ne génèrent pourtant pas d'anxiété [22,23]. Notre étude a d'ailleurs montré une surévaluation de l'anxiété par les professionnels. Cependant, les modalités de la communication sont déterminantes pour générer le moins d'angoisse possible [24,25]. Chez certains patients, la mise à distance protectrice vis-à-vis de l'évocation de la fin de vie, est un frein à l'appropriation des dispositifs. Chez les deux patients du groupe III, même si l'intervention d'information a eu un impact sur l'utilisation du dispositif, l'angoisse générée par les entretiens pose la question éthique du bénéfice pour le patient de la démarche d'information, exprimée par Mr $C$ : « Si les gens se sentent moins bien après qu'avant, cela sert à quoi ? ». L'intervention d'information ne devrait donc pas être proposée à ces patients qui ne souhaitent ni d'information pronostique ni de participation aux décisions. L'information donnée vient ici bousculer des mécanismes défensifs et compromettre l'adaptation à la réalité. [26] Cette constatation rejoint l'étude de Maciejewski et al. [27] qui montre que les discussions anticipées chez ces patients, peuvent paradoxalement induire un recours plus fréquent à des soins agressifs inappropriés en fin de vie.

Sollicités sur la personne qui doit effectuer la démarche, les patients de l'étude préfèrent que celle-ci ne soit pas directement impliquée dans sa prise en charge. Sur les 13 patients qui 
ont déjà évoqué leurs souhaits avant l'intervention, seul un en a parlé à son oncologue. La posture de l'oncologue est complexe [14] : parce qu'il véhicule l'espoir, les patients sont réticents à aborder les questions d'aggravation avec lui [8,28], néanmoins, il doit être au cœur du processus décisionnel et nécessairement impliqué dans les discussions anticipées $[29,30]$. Quant au meilleur moment désigné par les patients pour proposer l'intervention d'information, nos résultats convergent avec ceux de la littérature : plutôt au début d'une maladie grave, à un stade peu symptomatique de l'évolution de celle-ci, en tout cas, bien en amont de la phase avancée et/ou terminale [30]. Dans notre étude, le taux de survie à 4 mois est plus élevé chez les patients inclus, attestant du fait que l'intervention est intervenue plus précocement dans l'évolution de leur maladie. Un des éléments essentiel de la faisabilité de l'intervention est donc le moment auquel elle est proposée. Dans une cohorte américaine de décès de patients âgés de plus de 60 ans, les DA étaient rédigées en moyenne 20 mois avant le décès [31]. À partir d'entretiens avec des patients atteints de cancer pulmonaire ou digestif et ayant plus de 4 mois d'espérance de vie, Michael et al. décrivent l'initiation de discussions anticipées et l'élaboration de directives, comme un processus long et dynamique, à ajuster au gré de l'évolution de la maladie [32].

L'ensemble de nos résultats montre autant la faisabilité que la complexité de la démarche d'anticipation des souhaits en fin de vie, qui ne peut être réduite à l'utilisation des dispositifs légaux de la personne de confiance et des directives anticipées. Cette démarche doit s'intégrer dans un dispositif progressif et dynamique de discussions anticipées [24]. Une littérature abondante en décrit les modalités $[25,32,33]$ et montre leur impact sur la qualité des soins en fin de vie $[22,23,28]$. Ces travaux soulignent que la réalisation de ces discussions anticipées nécessitent un savoir-faire communicationnel, s'appuyant sur le maintien de l'incertitude [34], et sur un abord communicationnel du type " espérer le meilleur, se préparer au pire » [25].

La promotion des DA est renforcée dans le contexte français par le récent avis 121 du CCNE qui préconise que les DA deviennent " un véritable outil de dialogue avec le malade » [35]. Ce renforcement progressif du principe d'autonomie en France pose aussi la question de l'information des patients pour qu'ils puissent participer au processus décisionnel. Une information complète en phase avancée reste difficile à donner [36], le maintien de l'espoir étant connu comme un facteur essentiel chez les patients. Il est donc nécessaire de mener en France des études permettant d'évaluer les souhaits d'information pronostique des patients en oncologie et de poursuivre l'exploration des meilleures modalités d'information des patients sur de plus grands effectifs.

Déclaration d'intérêts : les auteurs déclarent ne pas avoir de conflits d'intérêts en relation avec cet article.

\section{Matériel complémentaire}

Le matériel complémentaire accompagnant la version en ligne de cet article est disponible sur http:// www.sciencedirect.com et doi:10.1016/j.bulcan.2015.01.009

Guide d'entretien 


\section{Références}

[1] Bouleuc C, Bredart A, Dolbeault S, Ganem G, Copel L. [How to improve cancer patients' satisfaction with medical information]. Bull Cancer (Paris) 2010;97(10):1173-81.

[2] Rapport d'information fait au nom de la mission d'évaluation de la loi nº 2005-370 du 22 avril 2005, relative aux droits des malades et à la fin de vie; et présenté par $M$. Jean Léonetti, député. Assemblée nationale. 2008 pp 305. Rapport $\Pi^{0} 1287$; 2008, Available from: http://www.assemblee-nationale.fr.

[3] Sicard D. Commission de réflexion sur la fin de vie en France. Penser solidairement la fin de vie. Rapport à François Hollande, président de la République Française; 2012;282, Available from: ladocumentationfrancaise.fr.

[4] Pennec S, Monnier A, Pontone S, Aubry R. End-of-life medical decisions in France: a death certificate follow-up survey 5 years after the 2005 Act of parliament on Patients' rights and End of life. BMC Palliat Care 2012;11(1):25

[5] Barbot A-C, Mussault P, Ingrand P, Tourani JM. Assessing 2-month clinical prognosis in hospitalized patients with advanced solid tumors. J Clin Oncol 2008;26(15):2538-43.

[6] Teno J, Lynn J, Wenger N, Phillips RS, Murphy DP, Connors AF, et al. Advance directives for seriously ill hospitalized patients: effectiveness with the patient self-determination act and the SUPPORT intervention. SUPPORT Investigators. Study to understand prognoses and preferences for outcomes and risks of treatment. J Am Geriatr Soc 1997;45 (4):500-7.

[7] Tan TS, Jatoi A. An update on advance directives in the medical record: findings from 1186 consecutive patients with unresectable exocrine pancreas cancer. J Gastrointest Cancer 2008;39(1-4):100-3.

[8] Lamont EB, Siegler M. Paradoxes in cancer patients' advance care planning. J Palliat Med 2000;3(1):27-35

[9] Lambert HC, McColl MA, Gilbert J, Wong J, Murray G, Shortt SED. Factors affecting longterm-care residents' decision-making processes as they formulate advance directives. Gerontologist 2005;45(5):626-33.

[10] Hanson LC, Tulsky JA, Danis M. Can clinical interventions change care at the end of life? Ann Intern Med 1997;126(5):381-8.

[11] Schwartz CE, Wheeler HB, Hammes B, Basque N, Edmunds J, Reed G, et al. Early intervention in planning end-of-life care with ambulatory geriatric patients: results of a pilot trial. Arch Intern Med 2002;162(14):1611-8.

[12] Billings JA, Bernacki R. Strategic targeting of advance care planning interventions: the
Goldilocks Phenomenon. JAMA Intern Med 2014;174(4):620.

[13] Vinant P, Bouleuc C. Directives anticipées: pour une meilleure qualité de la fin de vie? Rev Laennec 2014;3(62):43-56.

[14] Dow LA, Matsuyama RK, Ramakrishnan V, Kuhn L, Lamont EB, Lyckholm L, et al. Paradoxes in advance care planning: the complex relationship of oncology patients, their physicians, and advance medical directives. J Clin Oncol 2009;28(2):299-304.

[15] Thorevska N, Tilluckdharry L, Tickoo S, Havasi A, Amoateng-Adjepong $Y$, Manthous CA. Patients' understanding of advance directives and cardiopulmonary resuscitation. J Crit Care 2005:20(1):26-34.

[16] Mitchell AJ, Chan M, Bhatti H, Halton M, Grassi L, Johansen C, et al. Prevalence of depression, anxiety, and adjustment disorder in oncological, haematological, and palliativecare settings: a meta-analysis of 94 interview-based studies. Lancet Oncol 2011;12 (2): $160-74$

[17] Hagerty RG, Butow PN, Ellis PA, Lobb EA, Pendlebury S, Leighl N, et al. Cancer patient preferences for communication of prognosis in the metastatic setting. J Clin Oncol 2004;22 (9):1721-30.

[18] Paillaud E, Ferrand E, Lejonc J-L, Henry O, Bouillanne 0 , Montagne 0 . Medical information and surrogate designation: results of a prospective study in elderly hospitalised patients. Age Ageing 2007;36(3):274-9.

[19] Loi no 2002-303 du 4 mars 2002 relative aux droits des malades et à la qualité du système de santé. http://www.legifrance.gouv.fr/ (Dernier accès au site 13 fév 2015)

[20] Loi no 2005-370 du 22 avril 2005 relative aux droits des malades et à la fin de vie. http://www.legifrance.gouv.fr/ (Dernier accès au site 13 fév 2015).

[21] Azoulay E, Pochard F, Kentish-Barnes N, Chevret S, Aboab J, Adrie C, et al. Risk of post-traumatic stress symptoms in family members of intensive care unit patients. Am J Respir Crit Care Med 2005;171(9): 987-94.

[22] Wright AA, Zhang B, Ray A, MackJW, Trice E, Balboni T, et al. Associations between end-oflife discussions, patient mental health, medical care near death, and caregiver bereavement adjustment. JAMA 2008;300(14): 1665-73.

[23] Stein RA, Sharpe L, Bell ML, Boyle FM, Dunn SM, Clarke SJ. Randomized controlled trial of a structured intervention to facilitate end-oflife decision making in patients with advanced cancer. J Clin Oncol 2013;31 (27):3403-10.
[24] Tulsky JA. Beyond advance directives: importance of communication skills at the end of life. JAMA 2005;294(3):359-65.

[25] Back AL, Arnold RM, Quill TE. Hope for the best, and prepare for the worst. Ann Intern Med 2003;138(5):439-43.

[26] Ruszniewski M. Face à la maladie grave patients, familles, soignants. Dunod; 2004; 206.

[27] Maciejewski PK, Prigerson HG. Emotional numbness modifies the effect of end-of-life discussions on end-of-life care. J Pain Symptom Manage 2013;45(5):841-7.

[28] Mack JW, Cronin A, Keating NL, Taback N, Huskamp HA, Malin JL, et al. Associations between end-of-life discussion characteristics and care received near death: a prospective cohort study. J Clin Oncol 2012;30(35) 4387-95.

[29] Maltoni M, Scarpi E, Pittureri C, Martini F, Montanari L, Amaducci E, et al. Prospective Comparison of prognostic scores in palliative care cancer populations. Oncologist 2012;17 (3):446-54

[30] Levy MH, Adolph MD, Back A, Block S, Codada SN, Dalal S, et al. Palliative care. J Natl Compr Canc Netw 2012;10(10): 1284-309.

[31] Silveira MJ, Kim SY, Langa KM. Advance directives and outcomes of surrogate decision making before death. N Engl J Med 2010;362 (13):1211-8.

[32] Michael N, O'Callaghan C, Clayton J, Pollard A, Stepanov N, Spruyt 0, et al. Understanding how cancer patients actualise, relinquish, and reject advance care planning: implications for practice. Support Care Cancer 2013;21(8):2195-205.

[33] Clayton JM, Butow PN, Tattersall MHN, Devine RJ, Simpson JM, Aggarwal G, et al. Randomized controlled trial of a prompt list to help advanced cancer patients and their caregivers to ask questions about prognosis and end-of-life care. J Clin Oncol 2007;25 (6): 715-23

[34] Vinant P. L'annonce du pronostic en cancérologie. Fin de vie, éthique et société. Édition ERES: 2012

[35] Avis $n^{0} 121$. Fin de vie, autonomie de la personne volonté de mourir [Internet]; 2013 Available from: http://www. ccne-ethique.fr/sites/default/files/ publications/avis_121_0.pdf.

[36] Weeks IC, Catalano PJ, Cronin A, Finkelman MD, Mack JW, Keating NL, et al. Patients' expectations about effects of chemotherapy for advanced cancer. N Engl J Med 2012;367 (17):1616-25. 